\title{
Call for Papers: The Empirical Limits of Forensic Mental Health Assessment
}

Law and Human Behavior invites manuscript submissions for a special issue focused on the empirical limits of forensic mental health assessment. The strength of a field is in its awareness of its limits. Understanding and acknowledging what it does not know or cannot do increases its credibility regarding what it does know and can do. A realistic view of its limits creates the conditions necessary to develop a clear vision of the direction its research and development efforts need to take.

We encourage authors to submit reviews of a specific area of forensic mental health assessment that cover at minimum (a) what clinicians should be reasonably confident that they can do based on the empirical evidence, (b) what aspects of the assessment have little or no empirical foundation, (c) what steps practitioners should take in light of those values and limits, and (d) what research directions the field should take to seek empirical support for aspects of the evaluation that are not well supported. Limitations may take many forms, such as the longevity of clinical decisions and predictions or the legitimacy and usefulness of assessment tools.

Manuscripts reporting individual studies will be less appropriate than critical reviews or theoretical pieces. For example, a manuscript covering assessments about a defendant's Mental State at the Time of the Offense (insanity defense) might examine (a) the empirical evidence that clinicians can make diagnoses reliably, (b) evidence that they can make diagnoses retrospectively, (c) evidence that they can identify particular mental states related to those diagnostic conditions, and (d) what clinical and research methods have value for achieving these objectives. Authors may choose to write about any type of forensic mental health assessment; however, a few particularly relevant types stand out for us:

- Mental state at the time of offense (insanity defense)

- Sexual offender's likelihood of recidivism

- A child's or adolescent's likelihood for violence/recidivism

- Transfer (or waiver) to adult court

- A child's or adolescent's amenability to treatment

- Malingering

- Child custody evaluations

- Disability or mental injury 
The guest editors for this issue are Thomas Grisso, PhD, and Gina Vincent, PhD. Four copies of manuscripts, prepared for anonymous review, should be sent by November 1,2003 to

Thomas Grisso, $\mathrm{PhD}$

Forensic Training \& Research

Department of Psychiatry

University of Massachusetts Medical School

55 Lake Avenue North, WSH 8B-21

Worcester, Massachusetts 01655

Any questions should be directed to Dr Vincent at Gina.Vincent@umassmed.edu. 